\title{
La protection des ressources en eau minérale : éléments de réflexion
}

\author{
M. Lopoukhine \\ BRGM \\ Département Utilisation et Protection de l'espace géologique \\ Centre thématique eau minérale
}

\section{I ÉVOLUTION HISTORIQUE ET RÉGLE- MENTATION}

Le souci de protection des sources d'eau minérale apparaît vraisemblablement pour la première fois à la fin des années 1820, période connue sous le nom de guerre des sources, au cours de laquelle, notamment dans ce qui fut appelé ensuite le Bassin de Vichy, exploitants de sources et propriétaires de terrains limitrophes se livraient une véritable guerre, à coup de réalisations de nouveaux puits destinés à capter l'eau minérale, parfois au détriment du voisin.

Après de multiples hésitations, la Loi du 14 juillet 1856 instaure, pour ces eaux, la notion de source déclarée d'intérêt public et la possibilité de les protéger par la délimitation d'un périmètre de protection.

Cette mesure répondait aux objectifs de l'époque: à savoir, essentiellement, offrir aux exploitants pouvant faire la preuve réelle de leur activité, une protection contre le détournement de l'eau minérale qu'ils exploitaient par des concurrents.

Par contre, les eaux minérales étant réputées d'origine profonde (sinon mystérieuse : juvénile, etc.), ces mesures réglementaires n'expriment aucun souci de conservation de la qualité de l'eau des sources.

Il a donc fallu attendre 1937 puis surtout 1957 pour que soit introduite dans les textes la notion de périmètre sanitaire d'émergence qui, comme son nom l'indique, a pour objectif de préserver la qualité de la source en son point de captage, zone où l'eau minérale est particulièrement vulnérable aux atteintes à son environnement immédiat.

Enfin en 1992, la loi sur l'eau du 2 janvier traduit, dans les textes, la prise de conscience des effets possibles de la dégradation de l'environnement en surface sur la qualité des eaux minérales, à court comme à long terme ; elle modifie donc les articles du Code de la Santé Publique relatifs à la protection des eaux minérales en ouvrant la possibilité de réglementer travaux souterrains et activités de surface à l'intérieur de leur périmètre de protection.

\section{II $\square$ SITUATION ACTUELLE}

La France compte actuellement 104 sites où l'on exploite l'eau minérale en établissements thermaux et 54 sites où ces eaux sont conditionnées en bouteille avant d'être com-

The protection of mineral water resources : some considerations

The question of protecting mineral water resources is now under active discussion, with a view to changing the specific regulations applying to this type of water. In fact, although current regulations do provide for the possibility of establishing protective boundaries around mineral water springs, these measures, for partly historical reasons, are sometimes unable effectively to protect the quality of the water.

If protection is to be effective, it should no longer take into account the concept of a spring, but that of a reservoir, with all its complexity and diversity; it should also combine administrative measures - such as the control of surface activities - with others of a technical nature, such as adapting abstraction works to the geological features of each site.

The idea has also been put forward that in certain particular cases «natural hydrogeological parks » should be established, the aim being the long-term protection of certain mineral water resources having a particularly strong heritage value, by protecting the resource from any polluting activity. An attempt is made to evaluate the cost of such protection. 
mercialisées : sur cet ensemble, seuls une cinquantaine de sites ont fait l'objet de Déclaration d'Intérêt Public, assortie ou non de la définition d'un périmètre de protection.

Certains de ces périmètres sont " anecdotiques " : cercle de $150 \mathrm{~m}$ de rayon centré sur une source; d'autres sont illusoires : 2400 hectares englobant la totalité d'une agglomération d'environ 30000 habitants. Certains enfin sont plus adaptés car ayant fait l'objet de révisions périodiques au fur et à mesure de l'évolution des connaissances hydrogéologiques.

Par ailleurs, la plupart des sources comportent maintenant des périmètres sanitaires d'émergence, à l'exception toutefois de certaines d'entre elles exploitées sur la base d'autorisations anciennes.

\section{DE LA NOTION DE SOURCE À LA NO- TION DE GISEMENT}

II a fallu attendre la parution de la directive européenne du 15 juillet $1980\left(\mathrm{n}^{\circ} 80 / 777\right)$ concernant les eaux minérales pour voir enfin prise en compte dans ce texte réglementaire, la notion de gisement d'eau minérale.

"On entend par " eau minérale naturelle", une eau bactériologiquement saine, ayant pour origine une nappe ou un gisement souterrain et provenant d'une source exploitée par une ou plusieurs émergences naturelles ou forées.

L'eau minérale naturelle se distingue nettement de l'eau de boisson ordinaire :

- par sa nature, caractérisée par sa teneur en minéraux, oligo-éléments ou autres constituants et, le cas échéant, par certains effets ;

- par sa pureté originelle, l'une et l'autre caractéristiques ayant été conservées intactes en raison de l'origine souterraine de cette eau qui a été tenue à l'abri de tout risque de pollution ;

- la composition, la température et les autres caractéristiques essentielles de l'eau minérale doivent demeurer stables dans le cadre de fluctuations naturelles; en particulier, elles ne doivent pas être modifiées par les variations éventuelles de débit. ”

\section{Q QU'EST-CE QU'UN GISEMENT D'EAU MINÉRALE ?}

On peut proposer la définition suivante : «Un gisement d'eau minérale est l'ensemble de la structure géologique souterraine depuis la zone d'alimentation et jusqu'à la zone d'émergence, située au droit d'une zone géographique bien déterminée, et de laquelle il est possible d'extraire, moyennant la mise en œuvre de techniques appropriées, une eau souterraine de qualité déterminée, stable, reproductible, et identique, aux fluctuations naturelles près, à la qualité de la source le cas échéant, déjà agréée ».

On peut distinguer ainsi (fig. l) :

- en $A$, une aire d'alimentation avec infiltration d'eau météorique ;

- en B, un réseau d'infiltration ou colonne d'infiltration, vaste en volume, mais à vitesse d'écoulement très lente ;
- en C, un axe de collecte agissant en drain et permettant la remontée rapide de l'eau minérale.

Cette définition inclut l'ensemble des principes soustendant toute réflexion concernant la protection des eaux minérales :

- c'est l'ensemble de la ressource en eau minérale (gisement exploité par une ou plusieurs émergences naturelles ou forées) qui est considéré, et non plus la source ;

- l'eau est caractérisée par une qualité chimique particulière et constante ;

- elle doit être pure et bactériologiquement saine, naturellement ;

- ces caractéristiques doivent se pérenniser dans le temps en raison de l'origine souterraine de ces eaux qui doit leur conférer une protection naturelle contre tout risque de pollution.

La problématique de la protection des gisements et captages d'eaux minérales doit nécessairement être abordée en respectant le principe suivant : chaque ressource en eau minérale bénéficie d'une protection naturelle plus ou moins efficace selon les cas. Compte tenu de l'évolution des activités humaines de surface, qui génèrent un certain nombre de risques de pollution :

- comment apprécier le niveau de protection naturelle ?

- comment le renforcer?

\section{D COMMENT PROTÉGER LES RESSOUR- CES EN EAU MINÉRALE}

\subsection{Objectifs}

Il découle de ces considérations que l'objectif de protection doit englober :

- l'eau proprement dite avec un souci de préservation de la pérennité de ses qualités ;

- la structure géologique qui permet de conférer à cette eau son caractère d'eau minérale, et qui lui assure également un certain niveau de protection naturelle.

Cet objectif de protection doit par ailleurs être considéré aussi bien dans le court terme que dans le long terme.

\subsection{Moyens}

Trois types de protection doivent être combinés de façon aussi complémentaire que possible :

- la protection naturelle : conformément à l'esprit de la réglementation, elle doit être aussi efficace que possible sinon la plus efficace. En effet, les deux autres types de protection ne devraient que venir compléter et renforcer cette protection naturelle ; ce sont :

- les mesures de protection techniques destinées à renforcer la protection naturelle ;

- les mesures de protection de type administratif : à ne mettre en œuvre qu'en complément à la protection naturelle et aux mesures d'ordre technique qui la renforcent. 


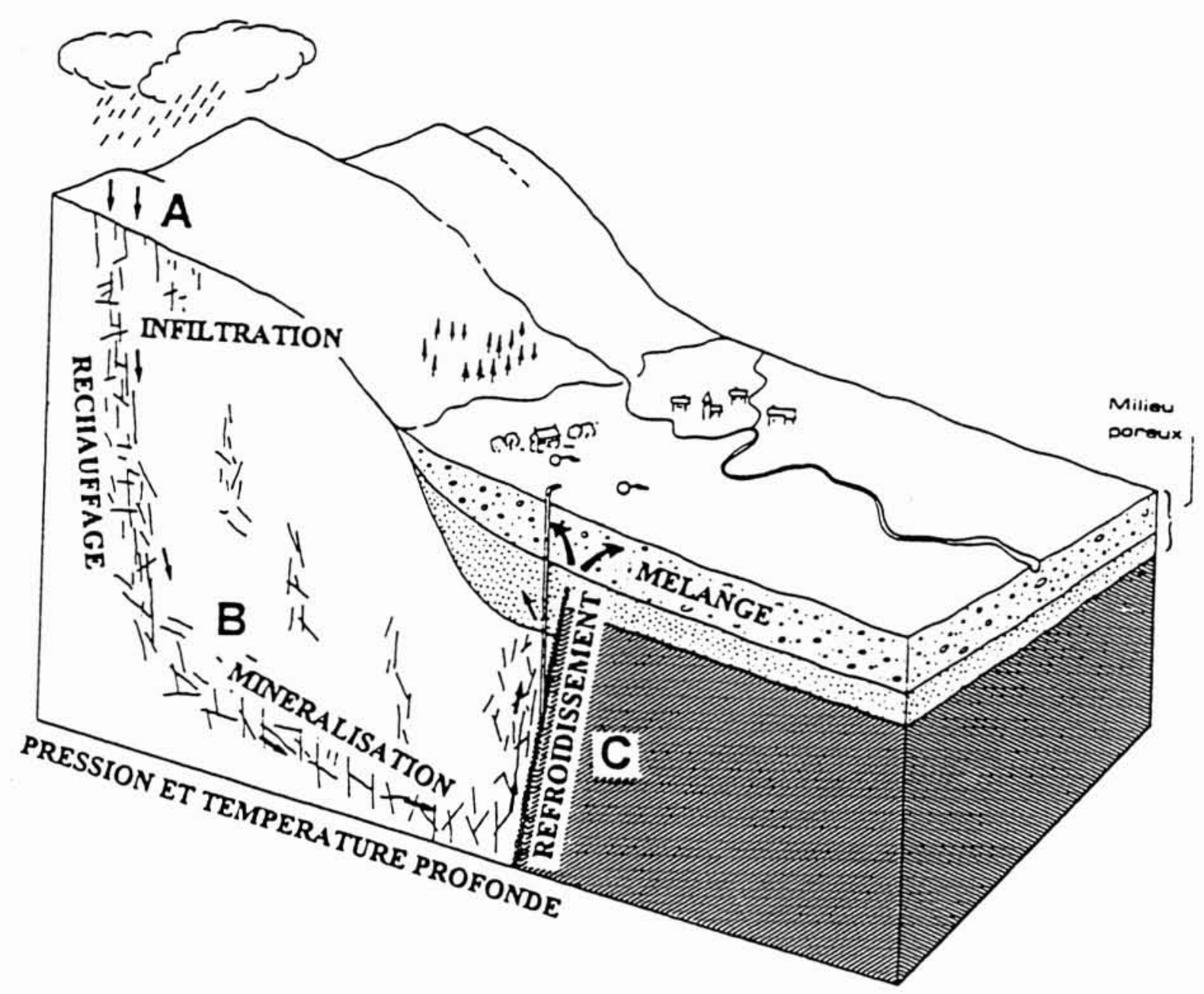

1. Les systèmes hydrothermaux.

\subsection{Lignes de conduite}

Chacune des zones A, B et C, constitutives du gisement va avoir, de par sa nature et son rôle dans le cycle de l'eau minérale, une sensibilité très différente vis-à-vis des risques envisageables.

La mise en place d'une démarche de protection du gisement devra donc faire appel à un «panachage» de mesures, différent pour chaque zone.

\subsubsection{Aire d'alimentation A}

L'aire d'alimentation des eaux minérales est par définition la seule portion du système qui soit totalement vulnérable ; il importe donc au plus haut point :

- de la définir et la circonscrire avec un maximum d'exactitude :

- de lui assurer une protection de type administratif, aussi complète que possible ;

- d'y mener des actions de préservation ou éventuellement de restauration de l'environnement naturel (notamment les forêts).

\subsubsection{Zone B}

La zone B ne peut être définie et circonscrite en soi. C'est la zone comprise entre alimentation $\mathrm{A}$ et zone d'émergence ou de captage $\mathrm{C}$.

C'est, par contre, la zone où en principe les conditions de protection naturelle sont les meilleures. Il conviendra donc d'en évaluer le niveau (hydrogéologie et connaissance du gisement), d'en apprécier éventuellement les points faibles et d'y pallier par des mesures techniques, ou/et par des mesures de type administratif.

\subsubsection{Zone d'émergence C}

Dans ce secteur, par définition, l'eau minérale emprunte un trajet qui la rapproche rapidement de la surface. L'efficacité de la protection naturelle de type hydrogéologique va donc s'en trouver diminuée. En outre, et c'est là l'une des conséquences historiques de l'application de la réglementation antérieure à 1957, les zones d'émergence ont été marquées par des développements urbains souvent importants, et ce parfois, en dépit de l'existence de périmètres de protection.

On devra donc dans cette zone, mettre en œuvre une protection technique aussi élaborée que possible. 


\begin{tabular}{|c|c|c|c|c|}
\hline & Zone A & Zone B & Zone C & \\
\hline $\begin{array}{l}\text { Mesures } \\
\text { administratives } \\
\text { (Etat, } \\
\text { collectivités) }\end{array}$ & & & & $\begin{array}{l}\text { Mesures techniques } \\
\text { (exploitant ou collec- } \\
\text { tivité) }\end{array}$ \\
\hline
\end{tabular}

2. Priorités à respecter pour un niveau de protection naturelle donné.

Cette protection consiste à réaliser le type de captage le mieux adapté à la géologie locale, de manière à ce que l'ensemble géologie locale plus captage constitue un tout, totalement étanche et permettant le captage de l'eau minérale à une profondeur telle, qu'elle puisse être amenée en surface, sans contact avec son environnement hydrogéologique, plus vulnérable à la pollution.

Les mesures de protection, de type administratif, ne devront être adoptées qu'en complément, avec les objectifs suivants :

- assurer la sécurité physique du captage,

- assurer le respect des conditions d'exploitation prescrites par les arrêtés d'autorisation,

- minimiser les atteintes à l'environnement, en relation avec l'urbanisation existante,

- limiter dans la mesure du possible l'urbanisation future, en fonction notamment des connaissances hydrogéologiques,

- restaurer progressivement un environnement de qualité.

Par contre, dans le cas d'exploitation d'eau minérale en voie de création, la protection technique devra être évi- demment assortie de mesures de protections administratives plus strictes, veillant cette fois-ci à préserver l'environnement pour l'avenir.

\section{VI $\square$ CONCLUSION}

La démarche de protection d'un gisement d'eau minérale ne peut être que globale; pour être applicable, elle doit s'appuyer sur un excellent niveau de connaissance technique de l'ensemble du gisement depuis l'aire d'alimentation jusqu'à la zone d'émergence.

Trois types de protection doivent être combinés :

- protection naturelle,

- protection technique,

- protection administrative.

Cette démarche de protection est de la responsabilité de trois intervenants différents :

- l'exploitant,

- la collectivité,

- l'Etat.

La philosophie de mise en œuvre de cette démarche de protection peut être résumée dans la figure 2. 